\title{
Behavior under impact of two polyvinyl acetate-polyethylene (PVA-PE) polymers and one elastomer - application to custom-made mouthguards
}

\author{
Philippe POISSON 1 , Philippe $\mathrm{VIOT}^{2}$ and Julien PETIT ${ }^{3}$ \\ ${ }^{1}$ Faculty of Dentistry, University of Victor Segalen Bordeaux 2, Bordeaux, France; Department of Dentistry and Oral Health, University Hospital, \\ Bordeaux, France; UMR 5227, University of Victor Segalen Bordeaux 2, Bordeaux, France \\ 2LAMEFIP, ENSAM de Bordeaux, Esplanade des Arts et Métiers, Talence, France \\ ${ }^{3}$ UMR 5227, University of Victor Segalen Bordeaux 2, Bordeaux, France \\ Corresponding author, Philippe POISSON; E-mail: philippe.poisson@u-bordeaux2.fr
}

\begin{abstract}
A drop tower was used to identify the mechanical behaviors of two polyvinyl acetate-polyethylene (PVA-PE) polymers and one elastomer used to fabricate custom-made mouthguards. The samples were in the form of either small cubes (edge length: $20 \mathrm{~mm}$ ) or large cubes (edge length: $30 \mathrm{~mm}$ ). Force and strain were recorded as functions of time, enabling a stressstrain analysis to be performed. In addition, a volume energy analysis was performed. While the three materials were viscoelastic, the stress-strain analysis showed that the PVA-PE polymers were more rigid than the elastomer, i.e., the same stress was reached with a lower strain. With the volume energy analysis, the two PVA-PE polymers were found to absorb a relatively large amount of energy with small sample deformations. Results of this study suggested that the mechanical properties of PVA-PE polymers are particularly useful and relevant for the fabrication of custom-made mouthguards.
\end{abstract}

Key words: Polyvinyl acetate-polyethylene (PVA-PE), Mouthguard materials, Impact absorption ability

Received Feb 3, 2008: Accepted Jul 8, 2008

\section{INTRODUCTION}

For prevention against dental injuries, the mouthguard seems to be the best preventive and protective measure ${ }^{1-4)}$. Presently, mouthguards are typically divided into three types: standard (type I), adaptable (type II), and custom-made (type III) ${ }^{5-9}$.

Amongst the three types currently available in the market, custom-made mouthguards offer the best possible fit and protection, and are hence the most effective means against dental injuries ${ }^{5,8-11)}$. To achieve a custom fit for maximum protection, the models of custom-made mouthguards are first fabricated according to specifications by a dentist. To shape the mouthguard material during model fabrication, plates or gutters are commonly used for thermoforming under high pressure or that cartridge are used to inject the material into the mold built from a cast of the patient's jaws.

In the case of orofacial and dental injuries, absorption is the most important factor involved in the damping of vibrations transmitted to the skull after a blow ${ }^{12}$. As such, this property of the materials used to make custom-made mouthguards must be optimized to fulfill the purpose of providing maximum protection. To date, a number of studies have been conducted on transmission of impact forces versus force absorption ${ }^{13-18)}$. It was found that polyvinyl acetate-polyethylene (PVA-PE) materials provided higher absorption than polyurethane and latex. Furthermore, the inclusion of air bubbles in the material lowered the transmission of the forces from an impact on the patient's jaw ${ }^{16,17)}$. At this juncture, it must be mentioned that these tests were conducted either on plates or mouthguards, whereby the latter were thermoformed either on a jaw model $^{19-21)}$ or on actual jaws ${ }^{12,22,23)}$.

Most of the studies measured the force transmitted by the materials as a function of time e $^{13,16-18,21,22)}$. However, the study of other material behaviors could be useful for mouthguard application. Therefore, the aim of this study was to analyze the mechanical properties of three materials used to make type III mouthguards, namely two PVA-PE polymers and one elastomer, in terms of transmitted force and impact energy absorption. Moreover, since the impact energy absorption behavior of these materials depends strongly on loading rates, it is necessary to assess this behavior under loading conditions close to those observed on the real structure.

\section{MATERIALS AND METHODS}

\section{Experimental samples}

All experimental samples were in cubic form. For each material, two edge lengths, $1_{c}{ }^{\prime}$ were used $(30 \pm 2$ $\mathrm{mm}$ and $20 \pm 2 \mathrm{~mm}$ ) to estimate the effect of sample size on impact energy absorption behavior of the material. The experimental approach was as follows. 


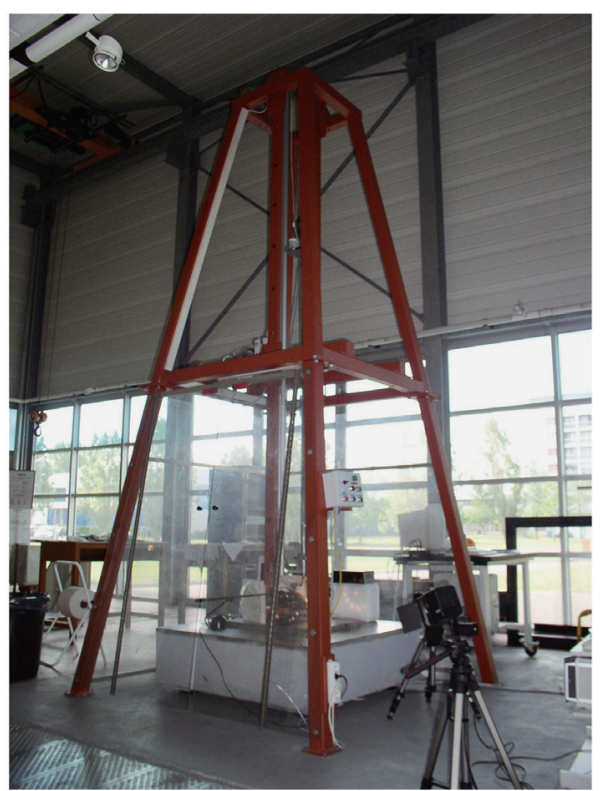

(a)

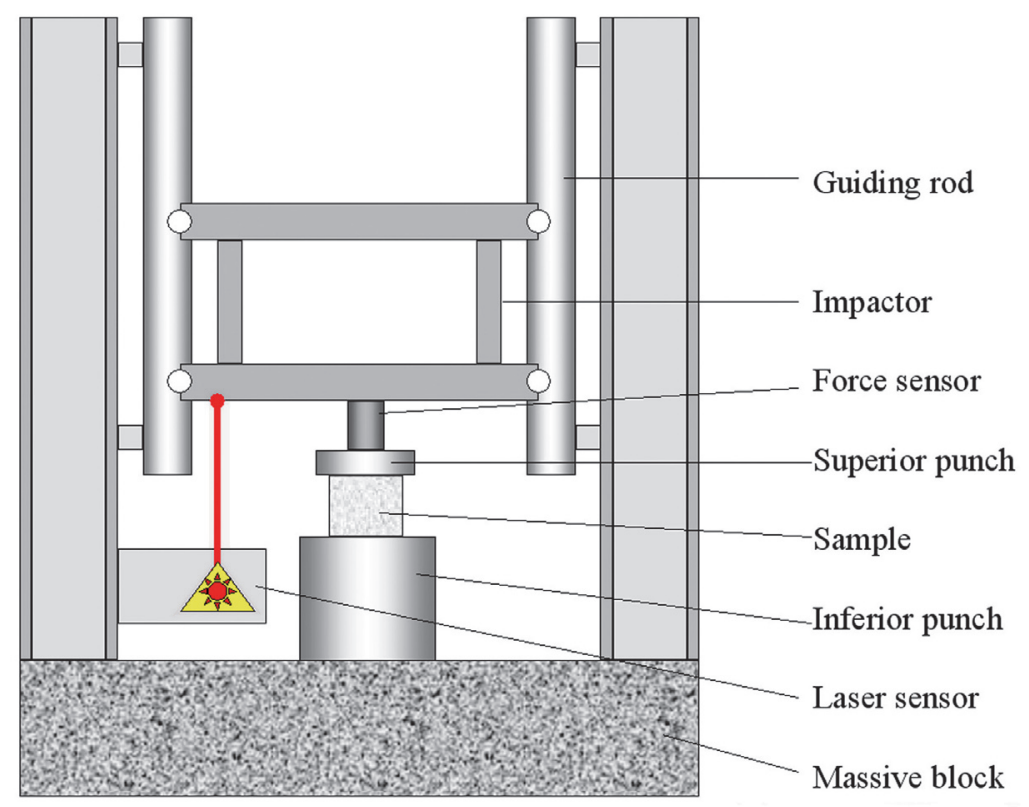

(b)

Fig. 1 (a) Drop tower apparatus; (b) Diagram of impact apparatus.

The true edge length $l_{c}$ of the cube (measurement accuracy of $0.02 \mathrm{~mm}$ ) was used to calculate the stress $\sigma$, whereby the same impact force applied to two different samples would then induce different stress values - and consequently different levels of strain. In this manner, the viscoelastic-plastic behavior of each material could be identified for two different values of compression stress from the same impact velocity condition.

For the first PVA-PE polymer, Bioplast (Scheu Dental GmbH, Iserlohn, Germany), the samples were thermoformed using several plates (thickness: $4 \mathrm{~mm}$ ). Therefore, the samples contained several layers separated by thin borders. These samples were named "Th" in this study.

For the second PVA-PE polymer, Corflex Orthodontic (Pressing Dental S.R.L., Dogana, San Marino), it was named "Co" in this study. As for the last material, SR Ivocap (Ivoclar Vivadent SAS, Saint-Jorioz, France), it was an elastomer made from acrylic resin and synthetic rubber and was named "El". Both Co and El samples were injected into molds and were therefore homogenous.

To simulate as close as possible the natural use of mouthguards, these samples were tested by applying an impact force using a drop tower.

\section{Impact apparatus: Drop Tower}

To characterize the materials used to fabricate mouthguards, it has to be done under loading conditions close to those observed on the structure. Mouthguards are subjected to dynamic loading, consequently causing compressive stress in their polymer structures. Against this background, dynamic compression was delivered on polymer samples in this study by applying a range of impact velocities close to those real-life shock loads experienced by mouthguards. To keep the simulation as close as possible to real-life loading on mouthguards, a drop tower was specially modified in this study to reproduce dynamic conditions of compression on these polymer samples. A drop tower was chosen in this study because it was a useful apparatus for material behavior identification to validate the properties and performance of mouthguard structures.

During a typical impact test on a drop tower, a projectile after release falls and strikes the specimen (sample or structure). During the drop, the velocity is chiefly determined by the drop height. Upon impact, the velocity depends on the response of the structure which absorbs and/or restitutes the impact energy because the projectile decelerates only during its penetration in the sample and then rebounds. Therefore, variations in projectile velocity allow the energy absorbed by the structure to be determined.

As for the modified drop tower used in this study, it consisted of two rectified columns attached to a metal gantry (Figs. 1a and 1b). These two columns served to guide the falling impactor. A winch (an electromagnet fixed to the projectile) was used to lift the projectile (mass: $1.8 \mathrm{~kg}$ ) to the desired impact height $(1 \mathrm{~m})$. In the present study, the theoretical velocity at impact was $\mathrm{V}_{\text {init }}=\sqrt{2 \cdot g \cdot h}$, 


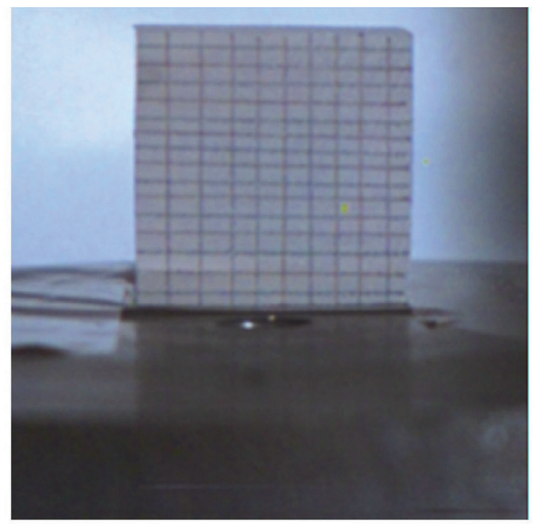

(a)

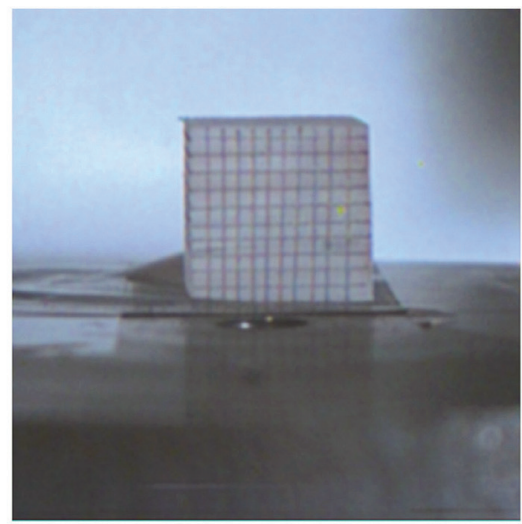

(c)

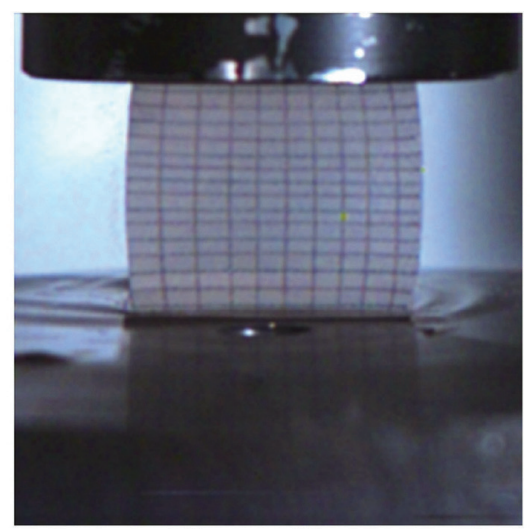

(b)

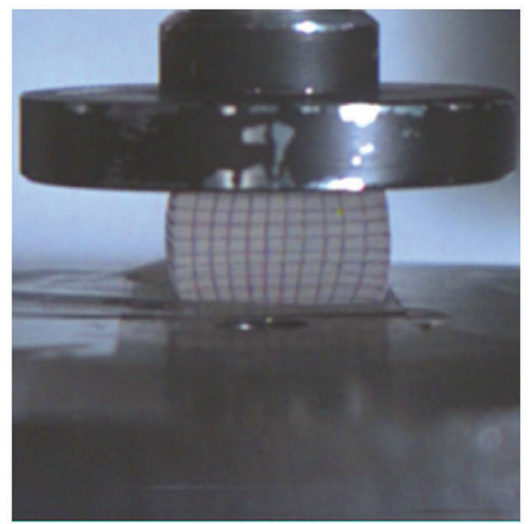

(d)

Fig. 2 (a) Large sample of Th (edge length: $30 \mathrm{~mm}$ ) before impact; (b) Large sample of Th during impact (photograph shows the maximum deformation); (c) Small sample of Th (edge length: $20 \mathrm{~mm}$ ) before impact; (d) Small sample of Th during impact (photograph shows the maximum deformation).

where $g$ was gravitational acceleration and $h$ was drop height. Based on this formula, the theoretical velocity for all tests in this study was $4.4 \mathrm{~m} \cdot \mathrm{s}^{-1}$ and the transmitted energy was $17.7 \mathrm{~J}$.

During the test, the projectile was released by the electromagnet and fell freely to strike the polymer sample. An anti-bouncing device was implemented to avoid a second shock which could further damage the structure. An analysis of the residual deformation and the damage was then performed. A laser sensor (effective range: $50 \mathrm{~mm}$ ) measured the impactor displacements during its fall, the impact phase and the rebound, thereby enabling the projectile velocity to be calculated. By placing a piezoelectric force sensor (Kistler Instrumente AG, Winterthur, Switzerland) under the sample, the force response of the sample during impact was also determined.

The signals of the two sensors were digitized and recorded by a data acquisition card (National Instruments) at a frequency of $100 \mathrm{KHz}$. A Phantom V4.0 high-speed camera (resolution: $256 \times 256$ pixels; speed: 3500 frames per second) was used to record the projectile-sample impact so as to enable a clear observation of sample deformation during impact and thus a better understanding of the test results. All tests were performed at room temperature $\left(24^{\circ} \mathrm{C}\right)$.

As for sample strain $\varepsilon$, it was calculated from the displacement $\Delta l$ of the impactor ( $\Delta l$ was null at the beginning of the impact) and the initial edge length $l_{c}$ of the sample as follows:

$$
\varepsilon=\ln \left(1-\frac{\Delta l}{l_{c}}\right)
$$

There were four samples for each material. The grid drawn on each sample (Figs $2 \mathrm{a}-\mathrm{d}$ ) was used to visualize the structure deformation and to determine the increase in sample section, which was needed for calculating the actual stress on the material.

After the impact, sample strain and force were recorded as functions of time to enable the identification of mechanical properties for each material. The video images obtained with the Phantom high-speed camera were therefore used to measure, with 


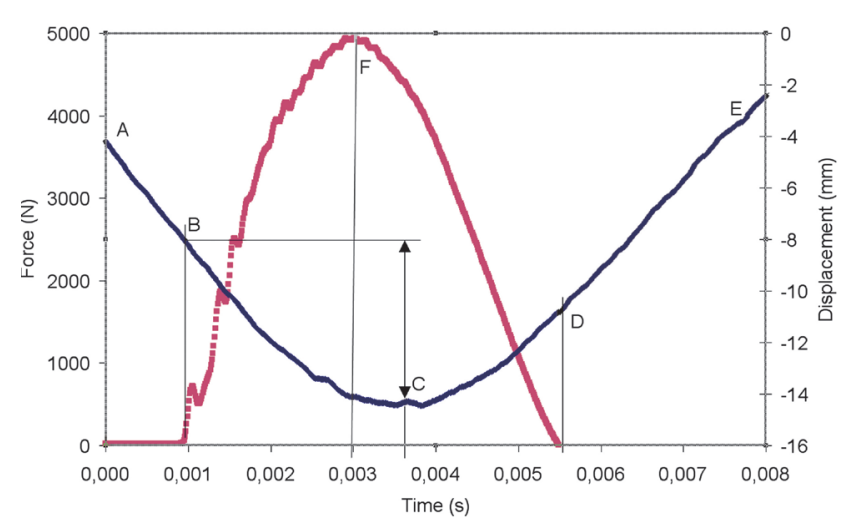

Fig. 3 Axial force (red curve) and displacement (dark blue curve) as functions of time (large sample of Co).

correlation techniques, the strain field of the material as a function of time ${ }^{24)}$.

\section{RESULTS}

Force and displacement vs. time results

By means of the drop tower, dynamic compressions were applied on two sample sizes of each of the three materials. Figures $2 \mathrm{a}$ and $2 \mathrm{~b}$ present, respectively, the $30-\mathrm{mm}$ cube before impact and at its maximum deformation during impact. Similarly, Figs. 2c and $2 \mathrm{~d}$ show the $20-\mathrm{mm}$ cube before impact and at its maximum deformation during impact. In the latter case of the smaller cube, it could be seen that it sustained a considerable deformation.

After calibration and conversion, the force (in Newtons) and displacement (in $\mathrm{mm}$ ) were recorded as functions of time. Figure 3 illustrates the force and displacement measurements, in red and dark blue curves respectively, for a large sample of Co. Sample loading actually began when the impactor came into contact with the sample, i.e., when the force began to increase (point B in Fig. 3 at $1 \mathrm{~ms}$ ). Before contact, the impactor displacement made it possible to verify the impactor speed which could be derived from the slope of the straight line $\mathrm{AB}$ (Fig. 3). After impact, the force increased considerably to reach a maximum (point $\mathrm{F}$ at $3 \mathrm{~ms}$ ). Oscillations in the force curve usually observed during an impact test were due to shock wave propagation in the drop tower and the material sample. During the compression stage, impactor velocity dropped substantially with its lowest at the minimum displacement value (time $=3.6$ $\mathrm{ms}$ at point $\mathrm{C}$ in Fig. 3). When impactor velocity became nil at point $\mathrm{C}$, the rebound began. During the rebound (after point $\mathrm{C}$ ), displacement velocity and the force decreased to reach values close to zero (point $\mathrm{D}$, time $=5.5 \mathrm{~ms}$ ). After point $\mathrm{D}$, the impactor

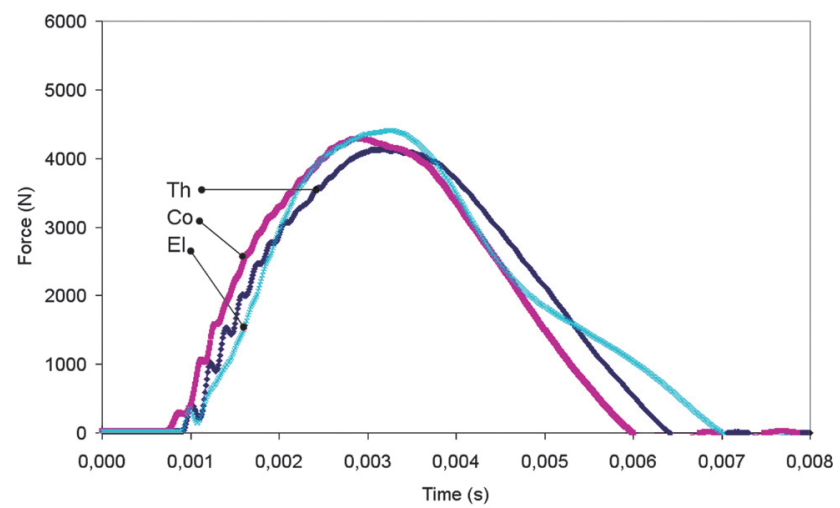

Fig. 4 Axial force as a function of time for the three tested materials.

was no longer in contact with the sample, hence enabling the slope of line DE to be used for final velocity calculation.

As for the energy absorbed by the sample during compression, it was estimated using the following formula:

$$
E=\frac{1}{2} \cdot m \cdot\left(\mathrm{V}_{f}^{2}-\mathrm{V}_{i}^{2}\right)
$$

where $V_{i}$ is the initial velocity and $V_{f}$ the final velocity.

Force vs. time results

As dynamic compressions were applied on the three materials' samples, the force measured during the test enabled the identification of each material's response under dynamic loading. Figure 4 shows the force response vs. time curves obtained in impacting the small cube sample of each material. The force response values obtained in Fig. 4 could be readily compared because all these samples had the same edge length $l_{c}$.

As seen in Fig. 4, the force response curves were very close. During the initial increase in force, the oscillations observed on the Th and Co polymers' curves were due to shock wave propagation in the materials' samples. However, no such oscillations appeared on the force response curve of $\mathrm{El}$, which meant that this material could be assumed to be sufficiently viscoelastic to absorb the shock wave. The shapes of the curves indicated that the three materials were fundamentally viscoelastic, whereby the El material appeared to have the highest viscoelasticity. On this note, the shape of the El curve was slightly different from those of Th and Co. At the beginning of the test with forces lower than 2500 $\mathrm{N}$, the time course of the force response for $\mathrm{El}$ was similar to those of $\mathrm{Th}$ and $\mathrm{Co}$, except that the 


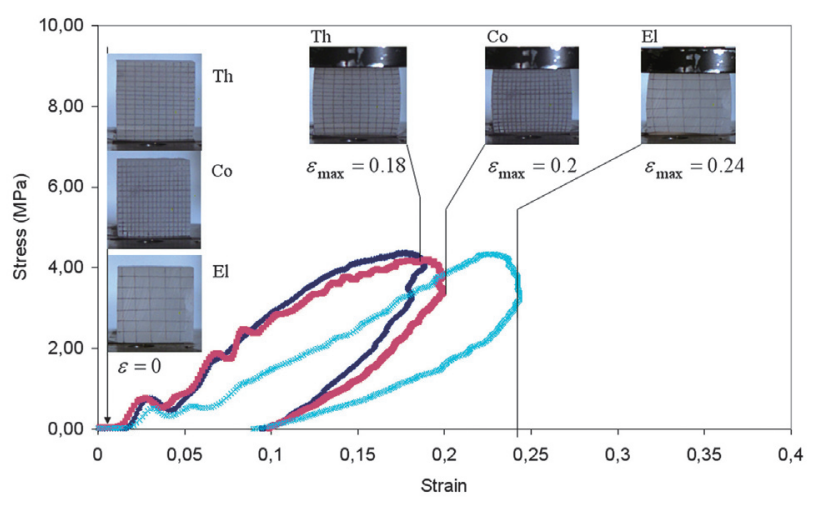

(a)

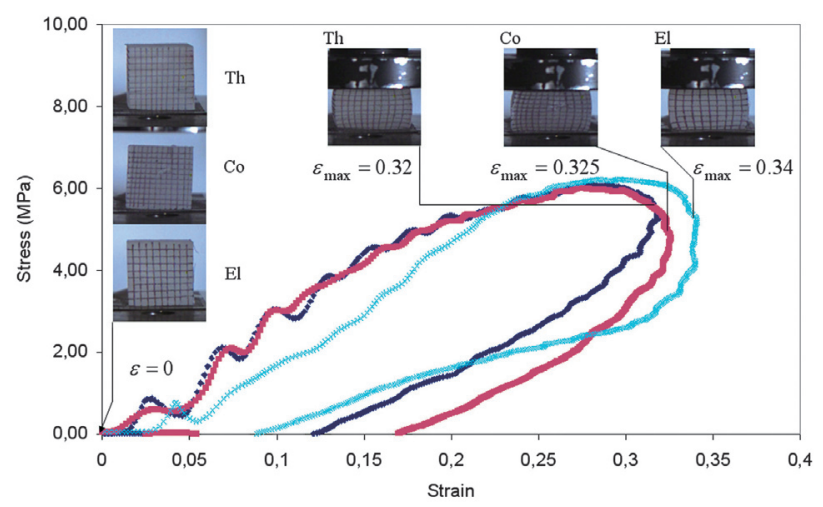

(b)

Fig. 5 (a) Axial stress as a function of axial strain for the three tested materials (large sample); (b) Axial stress as a function of axial strain for the three tested materials (small sample).

increase in force started after a small delay. At the end of the test, variation in force response for the El sample was slower than the other samples. Taken together, it could be said that the viscoelastic behaviors of these materials seemed slightly different.

\section{Stress vs. strain results}

The results obtained in Fig. 4, and hence the inferences made for the materials' viscoelastic behaviors, were confirmed by the stress response $\sigma$ of the materials as a function of strain $\varepsilon$ obtained for the large and small samples (Figs. 5a and 5b respectively). In addition, study of the changes in the shapes of the impacted samples using video images recorded with the high-speed camera would reveal interesting phenomena. Therefore in Figs. 5a and $5 \mathrm{~b}$, images of the large and small samples before impact (strain $\varepsilon=0$ ) and at the maximum strain are shown for all the three materials.

During dynamic compression, the stress-strain response curves (upper part) were almost the same for Th and Co, but that of the El material was somewhat different. Nonetheless, during this part of stress-strain response, these three materials could be considered to be elastic - or more precisely viscoelastic, because an influence of strain rate on their behaviors was suspected. Therefore, the curves could be approximated with a straight line until the strain reached 0.1 for Th and Co and 0.2 for El. The initial stiffness (estimated from the slope during the first part of the response) was similar for both Th and $\mathrm{Co}$, but which was two times greater than the El material. It was difficult to accurately determine the materials' elastic moduli from the dynamic tests because of strong perturbations generated by the impacts. On the other hand, it was conservatively safer to compare the relative values of stiffness.

The behavior, initially elastic, was again similar for both Th and Co polymers with a progressive decrease in curve slope. Conversely, for the El material, an increase in curve slope was observed. The maximum stress, $\sigma$, was approximately $6.2 \mathrm{MPa}$ for the three small material samples, but only 4.2 $\mathrm{MPa}$ for the large samples. Apparently, the dynamic compressions generated higher stresses in the small samples. This result was expected because when the same impact energy $\mathrm{E}$ was applied on the two different sample sizes, the volume energy calculated from the summation of stress and strain, $\mathrm{E}_{v}=\int \sigma . d \varepsilon$ ( $v=$ sample volume), was higher for the small samples. This higher volume energy then elicited higher stresses $\sigma$ and higher strains $\varepsilon$ in the small samples. Consequently, the greatest maximum strains (approximately 0.32) were obtained for the smaller samples (Fig. 5b). Indeed, this effect could be observed from the sample deformations captured on the video images (Figs. 5a and 5b). Furthermore, the highest strain value was obtained with the El material, at 0.24 for the large sample and 0.34 for the small sample. For Co, the maximum strain values were 0.2 for the large sample (Fig. 5a) and 0.325 for the small sample (Fig. 5b) - which were slightly higher than those of the Th samples.

The last part of the stress-strain response curve depicted the rebound of the impactor, and hence the unloading of the sample. The three stress values progressively dropped to zero (Fig. 5a) and a residual strain was observed - which was the same - for all the three large material samples, namely $\varepsilon_{\text {residual }}=0.1$. Volume energy was low for the large samples, and hence the damage induced by the impact (and consequently the residual strain) was accordingly small. However, such was not the case with the small samples (Fig. 5b). Whilst the residual strain for small El sample was still 0.1, the residual strain values were higher for the two polymers with Co reaching 0.17 for the small sample.

Energy vs. strain results

In this study, volume energy $\mathrm{E}_{v}=\int \sigma . d \varepsilon$ absorbed by 


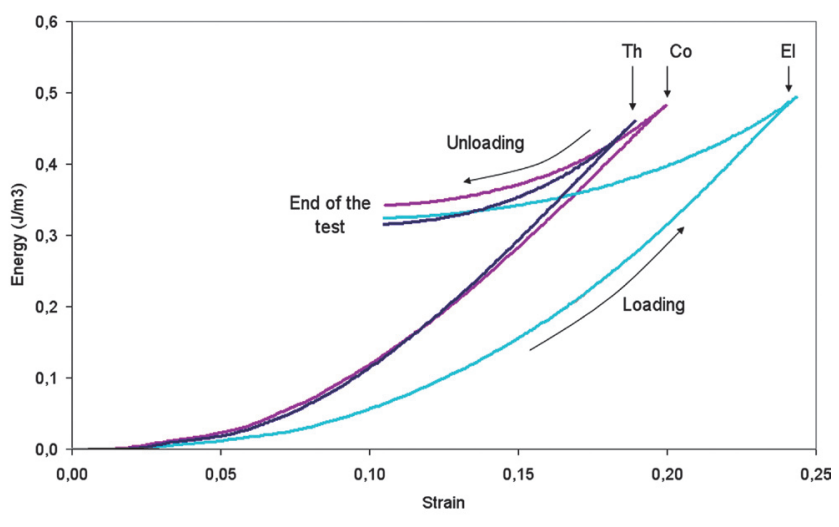

Fig. 6 Volume energy as a function of axial strain for the three tested materials (large sample).

the sample was calculated for each material and each sample size. However, only the results obtained from impacts carried out on large samples were used for analysis. Deformations of the large samples were relatively small as were the variations in the section of the sample normal to the load. These section variations, $\mathrm{S}$, were a classic defect of compression tests due to friction between the material sample and the punches. Mean stress was calculated as a ratio of the measured force to the mean section. A low variation in the $\mathrm{S}$ section of the large sample would therefore imply a relatively homogenous stress field, which made the calculation of volume energy more accurate.

Figure 6 shows the volume energy as a function of strain for the large samples of the three materials. At the beginning of the impact, the volume energy absorbed by the materials was small because stress and strain were low by virtue of their elastic behavior (for strain $\varepsilon<0.1$ ). During the second phase of loading where both stress and strain increased strongly, material behavior became viscoplastic and volume energy reached a value of approximately 0.49 $\mathrm{J} / \mathrm{m}^{3}$. The El material dissipated the highest volume energy - but at a higher strain value. For Th and Co polymers, they showed the same energetic behavior and attained similar values for their maximum volume energies $\left(0.46\right.$ and $0.48 \mathrm{~J} / \mathrm{m}^{3}$ respectively) - but at lower strain values. Finally, during the unloading phase, the volume energies absorbed by the samples decreased because part of that energy was transformed into impactor kinetic energy (hence the impactor rebounded and its velocity increased). At the end of the test, the final volume energy corresponded to the impact energy dissipated by the sample (by unit of volume). Amongst the three materials, Co attained the highest final volume energy at $\mathrm{E}_{\mathrm{v}}=0.34 \mathrm{~J} / \mathrm{m}^{3}$.

\section{DISCUSSION}

Increasingly stringent protection standards are required for safety equipment used in sports. To ensure conformance and credibility of standards for the end-users, the European Standardization Commission has specified test procedures and safety requirements pertaining to shock absorption or resistance to penetration. For shock resistance, a drop tower is usually used to perform the safety evaluation tests (Fig. 1a) ${ }^{25}$. Similarly in this study, this apparatus was used to identify the behaviors of materials under dynamic loading conditions similar to those imposed on mouthguards. Consequently, velocities observed during these impacts were indeed within the range of velocities observed for real-life shock loading on mouthguards. This experimental approach was necessary because the tested materials were viscoelastic in that their response in terms of force vs. time depended on the strain rate (induced by the projectile velocity).

These results can be analyzed from different points of view. From the industrial perspective, the behaviors of these elastomeric materials must be identified eventually to construct a model for the mouthguard using the Finite Element Method. From the clinical perspective, the ultimate objective is to optimize energy absorption by the mouthguard. This means that these materials must be classified in terms of impact energy absorption to limit the damage caused by the impact on the end-user.

\section{Material behavior}

In this study, it was shown that the three tested materials exhibited viscoplastic behavior under dynamic loading. This result was expected, but such characterization is usually done with quasi-static tests and rarely under dynamic loading conditions. Based on the impact results obtained in this study, the following arguments are hereby presented to confirm the viscoplastic behavior of the three tested materials. First, an analysis of the relations between force (or displacement) and time revealed that the minimum displacement of the projectile (point C, Fig. 3: maximum deformation of the sample) and the maximum force (point F, Fig. 3) did not appear at the same time. This was characteristic of the viscoplastic behavior of a polymer. Second, the variations in stress as a function of strain were different during the loading and unloading phases, which meant that a hysteresis was observed. This hysteresis occurrence was attributed to the viscoplastic behavior of the materials and the damage incurred due to dynamic loading.

Viscoplastic behavior has an influence on impact energy dissipation. An analysis of volume energy absorption revealed that the two PVA-PE polymers 
absorbed a significant amount of energy at low strain values and that the residual energy after impact was slightly higher for the Co material.

On the material damage incurred due to dynamic loading, it could be estimated from the residual strain for the three materials. Essentially, the residual strain levels were identical among the three materials at low volume energy. However, at higher volume energy, the damage to the $\mathrm{Th}$ and Co polymers increased significantly (hence inducing higher residual strains). This behavior rendered the materials capable of absorbing higher energy during shock impacts.

From the mechanical viewpoint, the abovementioned results efficaciously served the intended objective of material behavior identification in this study and were equally useful for parameter identification of a viscoplastic model (the latter phase of the study is in progress). In summary, the behavior of the three elastomeric materials was characterized and identified - by means of dynamic load testing using a drop tower - as follows:

(1) Variation of stress was determined as a function of strain. This macroscopic response would be useful to identifying the rheological model.

(2) Absorbed energy was quantified in per unit of material volume. This result would be useful to the classification of these elastomeric materials for passive safety related applications. Further, this result would enable the determination of mouthguard volume necessary to absorb real impact energy.

(3) Material damage was estimated from residual strain. This quantification method was of prime importance since it characterized the limits of each material in terms of impact energy dissipation.

\section{Clinical importance of the test results}

In the context of mouthguard applications, the product is deemed efficient if the force transmitted to the jaws is low and that the impact energy is largely absorbed. In this regard, the performance of mouthguard materials is classified according to the criteria of minimum transmitted force and maximum absorbed energy. Based on the results of this study, the Th polymer emerged as the best candidate with the minimum transmitted force of $4,130 \mathrm{~N}$. The force values recorded with the other two materials were higher at 4,280 $\mathrm{N}$ for Co and 4,400 $\mathrm{N}$ for El.

Analysis of the stress-strain behavior of the three materials corroborated the above conclusion. It was noted that both Th and Co polymers had a similar stress-strain behavior, whereby these materials were more rigid than $\mathrm{El}$ - that is, the same stress was reached with a lower strain. This meant that for mouthguard applications, the Th and Co materials are capable of withstanding higher stresses for lower strains. This finding is particularly important and relevant because a mouthguard has low thickness in many different parts, which means that deformations cannot be high.

On the second criterion about absorbed energy, analysis of the energy vs. strain response of the three materials showed that the $\mathrm{Th}$ and Co polymers exhibited a more favorable behavior. They absorbed a significant amount of energy with small sample deformations, a mechanical property which is highly essential for mouthguard applications.

\section{CONCLUSIONS}

To improve mouthguard efficiency, material response in terms of transmitted force on the jaws and impact energy absorption must be taken into account. In this study, these two criteria were used as means to classify the tested materials for their potential and performance as mouthguard materials.

Analysis of the mechanical properties revealed that the behaviors of Th and Co polymers under impact were particularly suitable for mouthguard applications. In terms of energy absorbed per unit volume, the El material presented the lowest absorbed energy whilst both Th and Co polymers had a similar response and absorbed approximately the same amount of energy. Between Th and Co polymers, Co had a slight upper-hand advantage in that it offered the maximum energy absorption.

This first step of the material behavior characterization study must be complemented and completed by an analysis of the responses of different mouthguards with different designs. To this end, a numerical approach could be employed to evaluate the energy absorption performances of different mouthguard structures arising from geometry and polymer type modifications. Further, this numerical approach would be needed to model the rheological behavior of polymers. Leveraging on the results of this study, it is now possible to develop the respective rheological models since the polymer properties have been dynamically identified.

\section{ACKNOWLEDGEMENTS}

The authors wish to thank Mr. Douglas GALLAGHER, translator, for his assistance in the use of English language for this paper.

\section{REFERENCES}

1) Chapman PJ, Nasser BP. Attitudes to mouthguards and prevalence of orofacial injuries in four teams competing at the second Rugby World Cup. Br J 
Sports Med 1993; 27: 197-199.

2) Chapman PJ. Mouthguards and the role of sporting team dentists. Aust Dent J 1989; 34: 36-43.

3) Onayeso CO, Adgbesan I. Oro-facial injury and mouthguard usage by athletes in Nigeria. Int Dent J 2003; 53: 231-236.

4) Woodmansey KF. Athletic mouthguards prevent orofacial injuries. J Am Coll Health 1997; 45: 179182.

5) Barnett F. Prevention of sports-related dental trauma: the role of mouthguards. Pract Proced Aesthet Dent 2003; 15: 391-394.

6) Chalmers DJ. Mouthguards: protection for the mouth in rugby union. Sports Med 1998; 25: 339349.

7) Newsome PRH, Tran DC, Cooke MS. The role of the mouthguard in the prevention of sports-related dental injuries : a review. Int J Paediatr Dent 2001; 11: 396-404.

8) Ranalli DN. Prevention of sports-related traumatic dental injuries. Dent Clin North Am 2000; 44: 3551 .

9) Scheer B. Prevention of dental and oral injuries. In: Textbook and color atlas of traumatic injuries to the teeth, $3^{\text {rd }}$ ed., Andreasen JO, Andreasen FM (eds), Munksgaard, Copenhagen, Denmark, 1994, pp.719-735.

10) Bass EH, Williams FA. A comparison of custom vs. standard mouth guards. A preliminary study. N Y State Dent J 1989; 55: 74-76.

11) Porter M, O'Brien M. The "Buy-Max" mouthguard: oral, peri-oral and cerebral protection for contact sports. J Iri Dent Assoc 1994; 40: 98-101.

12) Ou M, Taniguchi H, Ohyama T. Analysis on decay rate of vibration following impact to human dry skull with and without mouthguards. Bull Tokyo Med Dent Univ 1996; 43: 13-24.

13) Bulsara YR, Mathews IR. Forces transmitted through a laminated mouthguard material with a sorbothane insert. Endod Dent Traumatol 1998; 14: 45-47.
14) Takeda T, Ishigami $K$, Shintaro $K$, Nakajima $K$, Shimada, A, Regner CW. The influence of impact object characteristics on impact force and force absorption by mouthguard material. Dent Traumatol 2004; 20: 12-20.

15) Tran D, Cooke MS, Newsome PRH. Laboratory evaluation of mouthguard material. Dent Traumatol 2001; 17: 260-265.

16) Westerman B, Stringfellow PM, Eccleston JA. An improved mouthguard material. Aust Dent J 1997; 42: 189-191.

17) Westerman B, Stringfellow PM, Eccleston JA. Beneficial effects of air inclusions on the performance of ethylene vinyl acetate (EVA) mouthguard material. Br J Sports Med 2002; 36: 51-53.

18) Westerman B, Stringfellow PM, Eccleston JA. The effect on energy absorption of hard inserts in laminated EVA mouthguards. Aust Dent J 2000; 45: 21-23.

19) Bemelmanns P, Pfeiffer P. Shock absorption capacities of mouthguards in different types and thicknesses. Int J Sports Med 2001; 22: 149-153.

20) Greasley A, Imlach G, Karet B. Application of a standard test to the in vitro performance of mouthguards. Br J Sports Med 1998; 32: 17-19.

21) Westerman B, Stringfellow PM, Eccleston JA. EVA mouthguards: how thick should they be? Dent Traumatol 2002; 18: 24-27.

22) de Wet FA, Heyns M, Pretorius J. Shock absorption potential of different mouthguard materials. $\mathrm{J}$ Prosthet Dent 1999; 82: 301-306.

23) Morikawa M, Taniguchi H, Ohyama T. Evaluation of athletic mouthguard through vibration test on maxillary teeth of human dry skull. J Med Dent Sci 1998; 45: 9-18.

24) Viot P, Beani F, Lataillade JL. Polymeric foam behavior under dynamic compressive loading. J Mater Sci 2005; 40: 5829-5837.

25) Viot P. Polymer foams to optimize passive safety structures in helmets. Int J Crashworthiness 2007; 12: 299-310. 